\title{
Minimum Bit Error Rate Beamforming Receiver for Space-Division Multiple-Access Based Quadrature Amplitude Modulation Systems
}

\author{
Sheng $\mathrm{Chen}^{\dagger}$, Jiankang Zhang ${ }^{\dagger}$, Xiaomin $\mathrm{Mu}^{\ddagger}$ and Lajos $\mathrm{Hanzo}^{\dagger}$ \\ ${ }^{\dagger}$ School of Electronics and Computer Science, University of Southampton, Southampton SO17 1BJ, UK \\ ${ }^{\ddagger}$ School of Information Engineering, Zhengzhou University, Zhengzhou, China \\ E-mails: sqc@ecs.soton.ac.uk, jz09v@ecs.soton.ac.uk, iexmmu@zzu.edu.cn, lh@ecs.soton.ac.uk
}

\begin{abstract}
We considers the beamforming assisted multipleantenna receiver for space-division multiple-access based multiuser systems that employ high-throughput quadrature amplitude modulation (QAM) signalling. The bit error ratio (BER) expression as the function of the beamformer's weight vector is derived, and the minimum BER (MBER) beamforming receiver is then obtained as the solution of the resulting optimisation problem that minimises the MBER criterion. A simplified conjugate gradient algorithm, which has previously demonstrated its effectiveness in solving the minimum symbol error ratio (MSER) optimisation problem, is employed to solve this MBER optimisation. For highorder QAM, although the bit decision is an inherently more complicated procedure than making a symbol decision, it turns out that the computational complexity of computing the MBER solution is similar to that of computing the MSER solution. As expected, our simulation results show that both the MBER and MSER systems achieve the same BER performance, and they significantly outperform the standard minimum mean squares error based solution.
\end{abstract}

\section{INTRODUCTION}

The ever-increasing demand for mobile communication capacity has motivated the employment of space-division multiple access (SDMA) for the sake of improving the achievable spectral efficiency. A particular approach that has shown real promise in achieving substantial capacity enhancements is the use of adaptive beamforming receiver with antenna arrays [1]-[3]. Classically, beamforming design is based on minimising the mean square error (MSE) criterion. Since for a communication system, it is the achievable bit error ratio (BER), not the MSE performance, that really matters, the minimum BER (MBER) beamforming has been derived for binary phase shift keying (BPSK) systems [4] and quadrature phase shift keying (QPSK) systems [5]. Note that a QPSK system can be viewed as consisting of two BPSK systems. Quadrature amplitude modulation (QAM) schemes [6] have become popular in numerous wireless standards by virtue of providing a high throughput. Minimum symbol error ratio (MSER) beamforming receiver has been conceived for QAM systems [7].

To the best of our knowledge, however, no direct MBER solution has been derived for QAM systems to date. For a high-order QAM scheme, multiple bits that form a symbol point have different bit error probabilities and the bit decision procedure is a complicated one [6]. By contrast, making a symbol decision is easier, and it would appear that the symbol error ratio (SER) calculation therefore would be computationally less complex. This was partly the "reason" why the
MSER criterion was considered in our previous work [7], when studying high-order QAM systems. In this contribution, we explicitly derive the BER expression as the function of the beamformer's weight vector, and formulate the MBER beamforming for QAM systems as the solution of the resulting optimisation problem that minimises the MBER criterion. We adopt a computationally attractive simplified conjugate gradient algorithm, which has previously demonstrated its effectiveness in solving the MSER optimisation problem [7], to solve this MBER optimisation for SDMA based QAM beamforming systems. A surprising result of this study is that the complexity of the MBER optimisation turns out to be similar to that of the MSER optimisation. Moreover, our simulation results confirm that both the MBER and MSER systems have the same achievable BER performance, and they significantly outperform the standard minimum MSE (MMSE) based solution.

We adopt the following notational conventions in this contribution. Boldface capitals and lower-case letters stand for matrices and vectors, respectively, while $\mathbf{I}_{K}$ denotes the $K \times K$ identity matrix. Furthermore, $(\bullet)^{\mathrm{T}}$ and $(\bullet)^{\mathrm{H}}$ are the transpose and Hermitian operators, respectively, while $|\bullet|$ and $\|\bullet\|$ denote the magnitude and Euclidean norm operators, respectively. $E[\bullet]$ is the expectation operator, while $\Re[x]$ and $\Im[x]$ are the real and imaginary parts of $x$, respectively. Finally, $j=\sqrt{-1}$.

\section{SySTEM MODEL}

Consider the SDMA system that employs the $L$-element receive antenna array to support $M$ QAM users. The receive signal vector $\mathbf{x}(k)=\left[x_{1}(k) \cdots x_{L}(k)\right]^{\mathrm{T}}$ can be expressed as [3], [7]

$$
\mathbf{x}(k)=\mathbf{P b}(k)+\mathbf{n}(k)=\overline{\mathbf{x}}(k)+\mathbf{n}(k),
$$

where the Gaussian white noise vector $\mathbf{n}(k)=$ $\left[n_{1}(k) \cdots n_{L}(k)\right]^{\mathrm{T}}$ has a covariance matrix $E\left[\mathbf{n}(k) \mathbf{n}^{\mathrm{H}}(k)\right]=$ $2 \sigma_{n}^{2} \mathbf{I}_{L}, \mathbf{b}(k)=\left[b_{1}(k) \cdots b_{M}(k)\right]^{\mathrm{T}}$ is the transmitted symbol vector of the $M$ users, and the system matrix $\mathbf{P}$ is given by

$$
\mathbf{P}=\left[A_{1} \mathbf{s}_{1} \cdots A_{M} \mathbf{s}_{M}\right]=\left[\mathbf{p}_{1} \cdots \mathbf{p}_{M}\right]
$$

with $A_{i}$ being the $i$ th channel coefficient and the steering vector for user $i$ given by

$$
\mathbf{s}_{i}=\left[e^{j \omega_{c} t_{1}\left(\theta_{i}\right)} \cdots e^{j \omega_{c} t_{L}\left(\theta_{i}\right)}\right]^{\mathrm{T}} .
$$

In (3), $t_{l}\left(\theta_{i}\right)$ is the relative time delay at array element $l$ for user $i, \theta_{i}$ is the direction of arrival for user $i$, and $\omega_{c}=2 \pi f_{c}$ 
is the angular carrier frequency. We define the system's signal to noise ratio as $\mathrm{SNR}=\mathrm{E}_{\mathrm{b}} / \mathrm{N}_{\mathrm{o}}=\mathrm{E}_{\mathrm{b}} / 2 \sigma_{n}^{2}$, where $\mathrm{E}_{\mathrm{b}}$ is the average energy per bit of the QAM symbol.

For notational simplicity, we assume 16-QAM modulation. Therefore, the $k$ th transmitted symbol of user $i$, denoted as $b_{i}(k)$, takes the value from the symbol set

$$
\{ \pm 1 \pm j, \pm 1 \pm 3 j, \pm 3 \pm j, \pm 3 \pm 3 j\} .
$$

The approach adopted in this study, however, can be extended to higher-order QAM schemes. Without loss of generality, user 1 is assumed to be the desired user and the rest of the sources are the interfering users. A linear beamformer is employed, whose output is given by

$$
y(k)=\mathbf{w}^{\mathrm{H}} \mathbf{x}(k)=\mathbf{w}^{\mathrm{H}} \overline{\mathbf{x}}(k)+\mathbf{w}^{\mathrm{H}} \mathbf{n}(k)=\bar{y}(k)+e(k),
$$

where $\mathbf{w}=\left[w_{1} \cdots w_{L}\right]^{\mathrm{T}}$ is the beamformer weight vector, and $e(k)$ is Gaussian distributed with zero mean and $E\left[|e(k)|^{2}\right]=$ $2 \sigma_{n}^{2} \mathbf{w}^{\mathrm{H}} \mathbf{w}$. Define the combined impulse response of the beamformer and the system as $\mathrm{w}^{\mathrm{H}} \mathbf{P}=\left[c_{1} \cdots c_{M}\right]$. The beamformer's output can alternatively be expressed as

$$
y(k)=c_{1} b_{1}(k)+\sum_{i=2}^{M} c_{i} b_{i}(k)+e(k) .
$$

Denote $y_{R}(k)=\Re[y(k)]$ and $y_{I}(k)=\Im[y(k)]$. Provided that $c_{1}$ is real-valued and positive, the decisions regarding the two in-phase bits and two quadrature bits can be made separately based on $y_{R}(k)$ and $y_{I}(k)$, respectively, as defined in (7) and (8). If $c_{1}=\mathrm{w}^{\mathrm{H}} \mathbf{p}_{1}$ is complex-valued, the rotating operation $\mathbf{w}^{\text {new }}=\frac{c_{1}^{\text {old }}}{\left|c_{1}^{\text {ldd }}\right|} \mathbf{w}^{\text {old }}$ can be used to ensure a real and positive $c_{1}$. This rotation is a linear transformation and does not alter the achievable system's BER.

The in-phase bit combinations that map to the symbols $b_{R_{1}}(k)=\Re\left[b_{1}(k)\right]=-3,-1,1,3$ are $11,10,00,01$, and the two in-phase bits that form the real part of 16-QAM symbol are known as the in-phase class $1(\mathrm{C} 1)$ and class $2(\mathrm{C} 2)$ bits, respectively [6]. The decision for the in-phase $\mathrm{C} 1$ bit is given by

$$
\begin{cases}\mathrm{C} 1 \text { bit }=0, & \text { if } y_{R}(k)>0, \\ \mathrm{C} 1 \text { bit }=1, & \text { if } y_{R}(k) \leq 0,\end{cases}
$$

and the decision regarding the in-phase $\mathrm{C} 2$ bit is given by

$$
\begin{cases}\mathrm{C} 2 \mathrm{bit}=0, & \text { if }-2 c_{1}<y_{R}(k)<2 c_{1}, \\ \mathrm{C} 2 \mathrm{bit}=1, & \text { if } y_{R}(k) \leq-2 c_{1} \text { or } y_{R}(k) \geq 2 c_{1} .\end{cases}
$$

The decision rules for the quadrature $\mathrm{C} 1$ and $\mathrm{C} 2$ bits are given similarly based on $y_{I}(k)$. It is seen that this bit decision procedure is more complicated than making a symbol decision.

Classically, the beamformer's weight vector is determined by minimising the MSE metric of $E\left[\left|b_{1}(k)-y(k)\right|^{2}\right]$, which leads to the following MMSE solution [8]

$$
\mathbf{w}_{\text {MMSE }}=\left(\mathbf{P P}^{H}+\frac{2 \sigma_{n}^{2}}{\sigma_{b}^{2}} \mathbf{I}_{L}\right)^{-1} \mathbf{p}_{1},
$$

where $\sigma_{b}^{2}$ is the QAM symbol energy. The work [7] has derived the beamforming solution based on minimising the system's SER. The contribution of this study is to derive the MBER beamforming solution for SDMA based QAM systems.

\section{Minimum Bit Error Rate Beamforming}

The noise-free part of the beamformer input takes values from the finite set given by $\overline{\mathbf{x}}(k) \in \mathbb{X} \triangleq\left\{\overline{\mathbf{x}}^{(q)}=\mathbf{P b}^{(q)}, 1 \leq\right.$ $\left.q \leq N_{b}\right\}$, where $N_{b}=16^{M}$ and $\mathbf{b}^{(q)}, 1 \leq q \leq N_{b}$, are all the legitimate equiprobable sequences of $\mathbf{b}(k)$. Thus, the noisefree part of the beamformer output only takes values from the finite set given by $\bar{y}(k) \in \mathbb{Y} \triangleq\left\{\bar{y}^{(q)}=\mathbf{w}^{\mathrm{H}} \overline{\mathbf{x}}^{(q)}, 1 \leq q \leq N_{b}\right\}$. The real and imaginary parts of the set $\mathbb{Y}$ are respectively

$$
\left\{\begin{array}{l}
\mathbb{Y}_{R} \triangleq\left\{\bar{y}_{R}^{(q)}=\Re\left[\bar{y}^{(q)}\right], \bar{y}^{(q)} \in \mathbb{Y}\right\}, \\
\mathbb{Y}_{I} \triangleq\left\{\bar{y}_{I}^{(q)}=\Im\left[\bar{y}^{(q)}\right], \bar{y}^{(q)} \in \mathbb{Y}\right\} .
\end{array}\right.
$$

The set $\mathbb{Y}_{R}$ can be divided into the four conditioned subsets

$$
\left\{\begin{array}{c}
\mathbb{Y}_{R}^{( \pm 1)} \triangleq\left\{\bar{y}_{R}^{(q)} \in \mathbb{Y}_{R}: b_{R_{1}}(k)= \pm 1\right\}, \\
\mathbb{Y}_{R}^{( \pm 3)} \triangleq\left\{\bar{y}_{R}^{(q)} \in \mathbb{Y}_{R}: b_{R_{1}}(k)= \pm 3\right\} .
\end{array}\right.
$$

Similarly, $\mathbb{Y}_{I}$ can be partitioned into the four subsets $\mathbb{Y}_{I}^{( \pm 1)}$ and $\mathbb{Y}_{I}^{( \pm 3)}$, depending on the values of $b_{I_{1}}(k)$. The number of the points in each of these subsets is $N_{s b}=N_{b} / 4$.

The conditional PDF of $y_{R}(k)$ given $b_{R_{1}}(k)=+l$ is

$$
p\left(y_{R} \mid+l\right)=\frac{1}{N_{s b}} \sum_{\bar{y}_{R}^{(q)} \in \mathbb{Y}_{R}^{(+l)}} \frac{1}{\sqrt{2 \pi \sigma_{n}^{2} \mathbf{w}_{m}^{\mathrm{H}} \mathbf{w}_{m}}} e^{-\frac{\left(y_{R}-\bar{y}_{R}^{(q)}\right)^{2}}{2 \sigma_{n}^{2} \mathbf{w}_{m}^{\mathrm{H}} \mathbf{w}_{m}}}
$$

where $l=1,3$. We first show that only a quarter of $\mathbb{Y}_{R}^{(+l)}$ is really needed to define the conditional PDF $p\left(y_{R} \mid+l\right)$ by exploiting the shifting and symmetric distributing properties of the signal subsets

$$
\mathbb{Y}^{(l, i)} \triangleq\left\{\bar{y}^{(q)} \in \mathbb{Y}: b_{R_{1}}(k)=l, b_{I_{1}}(k)=i\right\},
$$

for $l, i=-3,-1,+1,+3$, as proved in [7]. Note that each $\mathbb{Y}^{(l, i)}$ contains $N_{\mathrm{sub}}=N_{b} / 16$ points. The real and imaginary parts of $\mathbb{Y}^{(l, i)}$ are given respectively by

$$
\left\{\begin{array}{c}
\mathbb{Y}_{R}^{(l, i)} \triangleq\left\{\bar{y}_{R}^{(q)}: \bar{y}^{(q)} \in \mathbb{Y}^{(l, i)}\right\}, \\
\mathbb{Y}_{I}^{(l, i)} \triangleq\left\{\bar{y}_{I}^{(q)}: \bar{y}^{(q)} \in \mathbb{Y}^{(l, i)}\right\},
\end{array}\right.
$$

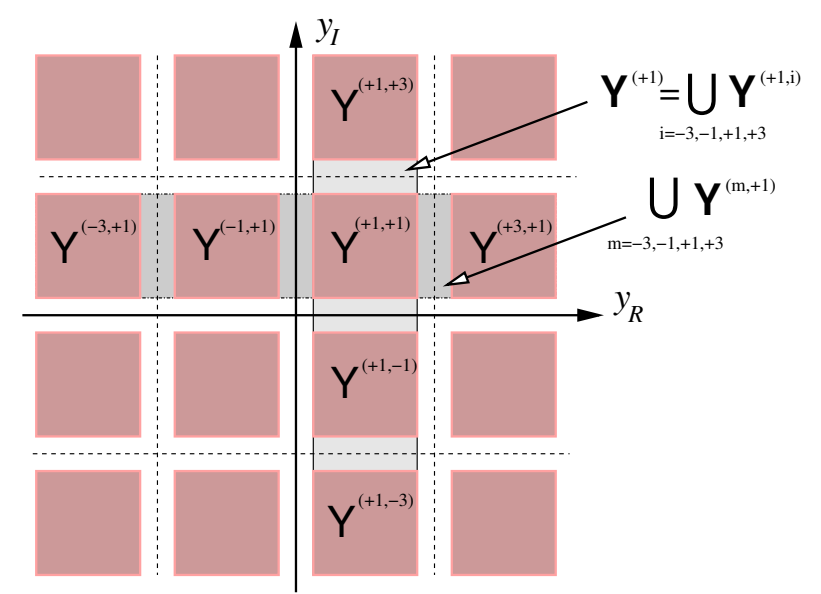

Fig. 1. Illustration of the shifting and symmetric distributing properties of the signal subsets, and the relationship $\mathbb{Y}^{(l)}=\bigcup_{i=-3,-1,+1,+3} \mathbb{Y}^{(l, i)}$. 
each containing $N_{\text {sub }}$ points. Noting the relationship

$$
\mathbb{Y}^{(l)}=\bigcup_{i=-3,-1,+1,+3} \mathbb{Y}^{(l, i)},
$$

as well as the shifting and symmetric distributing properties of $\mathbb{Y}^{(l, i)}$ as illustrated in Fig. 1, it then becomes clear that the real part of $\mathbb{Y}^{(l)}$, namely $\mathbb{Y}_{R}^{(+l)}$, is obtained by simply reproducing each signal point four times in $\mathbb{Y}_{R}^{(+l,+1)}$. Thus, the conditional PDF (12) can equivalently be expressed by

$p\left(y_{R} \mid+l\right)=\frac{1}{N_{\mathrm{sub}}} \sum_{\bar{y}_{R}^{(q)} \in \mathbb{Y}_{R}^{(+l,+1)}} \frac{1}{\sqrt{2 \pi \sigma_{n}^{2} \mathbf{w}_{m}^{\mathrm{H}} \mathbf{w}_{m}}} e^{-\frac{\left(y_{R}-\bar{y}_{R}^{(q)}\right)^{2}}{2 \sigma_{n}^{2} \mathbf{w}_{m}^{\mathrm{w}} \mathbf{w}_{m}}}$.

Taking into account the symmetric distribution of $\mathbb{Y}_{R}^{(+l,+1)}$ and $\mathbb{Y}_{R}^{(-l,+1)}$ with respect to the decision boundary $y_{R}=0$, the in-phase $\mathrm{C} 1$ bit error probability is given by

$$
\begin{aligned}
P_{E_{R}, C 1}(\mathbf{w})= & \frac{1}{2 N_{\mathrm{sub}}}\left(\sum_{\bar{y}_{R}^{(q)} \in \mathbb{Y}_{R}^{(+1,+1)}} Q\left(g_{R}^{(q)}(\mathbf{w})\right)\right. \\
& \left.+\sum_{\bar{y}_{R}^{(q)} \in \mathbb{Y}_{R}^{(+3,+1)}} Q\left(g_{R}^{(q)}(\mathbf{w})\right)\right),
\end{aligned}
$$

where $Q(u)=\frac{1}{\sqrt{2 \pi}} \int_{u}^{\infty} e^{-\frac{v^{2}}{2}} d v$,

$$
g_{R}^{(q)}(\mathbf{w})=\frac{\operatorname{sgn}\left(\Re\left[b_{1}^{(q)}\right]\right) \bar{y}_{R}^{(q)}}{\sigma_{n} \sqrt{\mathbf{w}^{H} \mathbf{w}}}=\frac{\operatorname{sgn}\left(b_{R_{1}}^{(q)}\right) \Re\left[\mathbf{w}^{H} \overline{\mathbf{x}}^{(q)}\right]}{\sigma_{n} \sqrt{\mathbf{w}^{H} \mathbf{w}}},
$$

and $b_{1}^{(q)}$ denotes the first element of $\mathbf{b}^{(q)}$, corresponding to the desired user's symbol $b_{1}(k)$. Noting the shift property $\mathbb{Y}_{R}^{(+3,+1)}=2 c_{1}+\mathbb{Y}_{R}^{(+1,+1)}$ again, $P_{E_{R}, C 1}(\mathbf{w})$ can be simplified as

$P_{E_{R}, C 1}(\mathbf{w})=\frac{1}{2 N_{\mathrm{sub}}} \sum_{\bar{y}_{R}^{(q)} \in \mathbb{Y}_{R}^{(+1,+1)}}\left(Q\left(g_{R}^{(q)}(\mathbf{w})\right)+Q\left(g_{R}^{(q, a)}(\mathbf{w})\right)\right)$,

where

$$
g_{R}^{(q, a)}(\mathbf{w})=\frac{2 c_{1}+\operatorname{sgn}\left(b_{R_{1}}^{(q)}\right) \bar{y}_{R}^{(q)}}{\sigma_{n} \sqrt{\mathbf{w}^{H} \mathbf{w}}} .
$$

Similarly, the quadrature $\mathrm{C} 1$ bit error probability is given by $P_{E_{I}, C 1}(\mathbf{w})=\frac{1}{2 N_{\mathrm{sub}^{(q)}}} \sum_{\bar{y}_{I}^{(q)} \in \mathbb{Y}_{I}^{(+1,+1)}}\left(Q\left(g_{I}^{(q)}(\mathbf{w})\right)+Q\left(g_{I}^{(q, a)}(\mathbf{w})\right)\right)$,

with

$$
\begin{gathered}
g_{I}^{(q)}(\mathbf{w})=\frac{\operatorname{sgn}\left(\Im\left[b_{1}^{(q)}\right]\right) \bar{y}_{I}^{(q)}}{\sigma_{n} \sqrt{\mathbf{w}^{H} \mathbf{w}}}=\frac{\operatorname{sgn}\left(b_{I_{1}}^{(q)}\right) \Im\left[\mathbf{w}^{H} \overline{\mathbf{x}}^{(q)}\right]}{\sigma_{n} \sqrt{\mathbf{w}^{H} \mathbf{w}}}, \\
g_{I}^{(q, a)}(\mathbf{w})=\frac{2 c_{1}+\operatorname{sgn}\left(b_{I_{1}}^{(q)}\right) \bar{y}_{I}^{(q)}}{\sigma_{n} \sqrt{\mathbf{w}^{H} \mathbf{w}}} .
\end{gathered}
$$

The $\mathrm{C} 2$ bit error probability is more involved. Consider the conditional in-phase C2 BER given $b_{R_{1}}(k)=+1$ first, which can be shown to be

$$
\begin{aligned}
P_{E_{R}, C 2}^{(+1)}(\mathbf{w})= & \frac{1}{N_{\mathrm{sub}}} \sum_{\bar{y}_{R}^{(q)} \in \mathbb{Y}_{R}^{(+1,+1)}}\left(Q\left(g_{R}^{(q, a)}(\mathbf{w})\right)\right. \\
& \left.+Q\left(g_{R}^{(q, b)}(\mathbf{w})\right)\right)
\end{aligned}
$$

with

$$
g_{R}^{(q, b)}(\mathbf{w})=\frac{2 c_{1}-\operatorname{sgn}\left(b_{R_{1}}^{(q)}\right) \bar{y}_{R}^{(q)}}{\sigma_{n} \sqrt{\mathbf{w}^{H} \mathbf{w}}} .
$$

However, the symmetric distributing property of $\mathbb{Y}_{R}^{(+1,+1)}$ implies that

$$
\sum_{\bar{y}_{R}^{(q)} \in \mathbb{Y}_{R}^{(+1,+1)}} Q\left(g_{R}^{(q, b)}(\mathbf{w})\right)=\sum_{\bar{y}_{R}^{(q)} \in \mathbb{Y}_{R}^{(+1,+1)}} Q\left(g_{R}^{(q)}(\mathbf{w})\right)
$$

and, therefore, $P_{E_{R}, C 2}^{(+1)}(\mathbf{w})$ can alternatively be expressed as

$$
\begin{aligned}
P_{E_{R}, C 2}^{(+1)}(\mathbf{w})= & \frac{1}{N_{\text {sub }}} \sum_{\bar{y}_{R}^{(q)} \in \mathbb{Y}_{R}^{(+1,+1)}}\left(Q\left(g_{R}^{(q)}(\mathbf{w})\right)\right. \\
& \left.+Q\left(g_{R}^{(q, a)}(\mathbf{w})\right)\right) .
\end{aligned}
$$

The conditional in-phase $\mathrm{C} 2 \mathrm{BER}$ given $b_{R_{1}}(k)=+3$ can be shown to be

$$
\begin{aligned}
P_{E_{R}, C 2}^{(+3)}(\mathbf{w})= & \frac{1}{N_{\mathrm{sub}}} \sum_{\bar{y}_{R}^{(q)} \in \mathbb{Y}_{R}^{(+3,+1)}}\left(Q\left(g_{R}^{(q, c)}(\mathbf{w})\right)\right. \\
& \left.-Q\left(g_{R}^{(q, a)}(\mathbf{w})\right)\right),
\end{aligned}
$$

where

$$
g_{R}^{(q, c)}(\mathbf{w})=\frac{\operatorname{sgn}\left(b_{R_{1}}^{(q)}\right) \bar{y}_{R}^{(q)}-2 c_{1}}{\sigma_{n} \sqrt{\mathbf{w}^{H} \mathbf{w}}} .
$$

The shifting properties of the signal subsets again imply that

$$
\sum_{\bar{y}_{R}^{(q)} \in \mathbb{Y}_{R}^{(+3,+1)}} Q\left(g_{R}^{(q, c)}(\mathbf{w})\right)=\sum_{\bar{y}_{R}^{(q)} \in \mathbb{Y}_{R}^{(+1,+1)}} Q\left(g_{R}^{(q)}(\mathbf{w})\right),
$$

while the second summary term in (27) is much smaller than any other summation term in the BER expressions (19), (26) and (27). Thus, $P_{E_{R}, C 2}^{(+3)}(\mathbf{w})$ can be simplified as

$$
P_{E_{R}, C 2}^{(+3)}(\mathbf{w})=\frac{1}{N_{\mathrm{sub}}} \sum_{\bar{y}_{R}^{(q)} \in \mathbb{Y}_{R}^{(+1,+1)}} Q\left(g_{R}^{(q)}(\mathbf{w})\right) .
$$

The in-phase $\mathrm{C} 2$ bit error probability is therefore given by

$$
\begin{aligned}
& P_{E_{R}, C 2}(\mathbf{w})=\frac{1}{2}\left(P_{E_{R}, C 2}^{(+1)}(\mathbf{w})+P_{E_{R}, C 2}^{(+3)}(\mathbf{w})\right) \\
& =\frac{1}{2 N_{\mathrm{sub}}} \sum_{\bar{y}_{R}^{(q)} \in \mathbb{Y}_{R}^{(+1,+1)}}\left(2 Q\left(g_{R}^{(q)}(\mathbf{w})\right)+Q\left(g_{R}^{(q, a)}(\mathbf{w})\right)\right) .
\end{aligned}
$$

Similarly, the quadrature $\mathrm{C} 2$ bit error probability is given by

$$
\begin{aligned}
P_{E_{I}, C 2}(\mathbf{w})= & \frac{1}{2 N_{\mathrm{sub}}} \sum_{\bar{y}_{I}^{(q)} \in \mathbb{Y}_{I}^{(+1,+1)}}\left(2 Q\left(g_{I}^{(q)}(\mathbf{w})\right)\right. \\
& \left.+Q\left(g_{I}^{(q, a)}(\mathbf{w})\right)\right) .
\end{aligned}
$$


The BER of the 16-QAM beamformer with weight vector $\mathbf{w}$ is therefore given by

$$
\begin{aligned}
& P_{E}(\mathbf{w})=\frac{P_{E_{R}, C 1}(\mathbf{w})+P_{E_{I}, C 1}(\mathbf{w})+P_{E_{R}, C 2}(\mathbf{w})+P_{E_{I}, C 2}(\mathbf{w})}{4} \\
& =\frac{1}{4 N_{\mathrm{sub}}}\left(\sum_{\bar{y}_{R}^{(q)} \in \mathbb{Y}_{R}^{(+1,+1)}}\left(\frac{3}{2} Q\left(g_{R}^{(q)}(\mathbf{w})\right)+Q\left(g_{R}^{(q, a)}(\mathbf{w})\right)\right)\right. \\
& \left.+\sum_{\bar{y}_{I}^{(q)} \in \mathbb{Y}_{I}^{(+1,+1)}}\left(\frac{3}{2} Q\left(g_{I}^{(q)}(\mathbf{w})\right)+Q\left(g_{I}^{(q, a)}(\mathbf{w})\right)\right)\right)
\end{aligned}
$$

The MBER beamformer solution is defined as

$$
\mathbf{w}_{\text {MBER }}=\arg \min _{\mathbf{w}} P_{E}(\mathbf{w}) .
$$

By comparing the BER expression (32) with the SER expression of [7], it is interesting to see that the BER calculation turns out to require a similar computational complexity as that of the SER calculation, at least for the 16-QAM case. Specifically, both the BER and SER can be computed based only on the single signal subset $\mathbb{Y}^{(+1,+1)}$. The MBER optimisation defined in (33) therefore has similar computational requirements as the MSER optimisation derived in [7]. This is surprising, since the bit decision procedure is inherently more complicated than the symbol decision procedure.

\section{GRadient Optimisation BAsed MBER BEAMFORMING}

The gradient of $P_{E}(\mathbf{w})$ with respect to $\mathbf{w}$ is

$$
\begin{aligned}
& \nabla P_{E}(\mathbf{w})=\frac{1}{4 N_{\mathrm{sub}}}\left(\sum _ { \overline { y } _ { R } ^ { ( q ) } \in \mathbb { Y } _ { R } ^ { ( + 1 , + 1 ) } } \left(\frac{3}{2} \nabla Q\left(g_{R}^{(q)}(\mathbf{w})\right)\right.\right. \\
& \left.+\nabla Q\left(g_{R}^{(q, a)}(\mathbf{w})\right)\right)+\sum_{\bar{y}_{I}^{(q)} \in \mathbb{Y}_{I}^{(+1,+1)}}\left(\frac{3}{2} \nabla Q\left(g_{I}^{(q)}(\mathbf{w})\right)\right. \\
& \left.\left.+\nabla Q\left(g_{I}^{(q, a)}(\mathbf{w})\right)\right)\right)
\end{aligned}
$$

with

$$
\nabla Q\left(g_{R}^{(q)}(\mathbf{w})\right)=\frac{e^{-\frac{1}{2}\left(g_{R}^{(q)}(\mathbf{w})\right)^{2}} \operatorname{sgn}\left(b_{R_{1}}^{(q)}\right)}{2 \sqrt{2 \pi} \sigma_{n} \sqrt{\mathbf{w}^{\mathrm{H}} \mathbf{w}}}\left(\frac{\bar{y}_{R}^{(q)} \mathbf{w}}{\mathbf{w}^{\mathrm{H}} \mathbf{w}}-\overline{\mathbf{x}}^{(q)}\right),
$$

$$
\begin{aligned}
\nabla Q\left(g_{R}^{(q, a)}(\mathbf{w})\right)= & \frac{e^{-\frac{1}{2}\left(g_{R}^{(q, a)}(\mathbf{w})\right)^{2}}}{2 \sqrt{2 \pi} \sigma_{n} \sqrt{\mathbf{w}^{\mathrm{H}} \mathbf{w}}}\left(\frac{2 c_{1}+\operatorname{sgn}\left(b_{R_{1}}^{(q)}\right) \bar{y}_{R}^{(q)}}{\mathbf{w}^{\mathrm{H}} \mathbf{w}} \mathbf{w}\right. \\
& \left.-\operatorname{sgn}\left(b_{R_{1}}^{(q)}\right) \mathbf{x}^{(q)}-2 \mathbf{p}_{1}\right), \\
\nabla Q\left(g_{I}^{(q)}(\mathbf{w})\right)= & \frac{e^{-\frac{1}{2}\left(g_{I}^{(q)}(\mathbf{w})\right)^{2}} \operatorname{sgn}\left(b_{I_{1}}^{(q)}\right)}{2 \sqrt{2 \pi} \sigma_{n} \sqrt{\mathbf{w}^{\mathrm{H}} \mathbf{w}}}\left(\frac{\bar{y}_{I}^{(q)} \mathbf{w}}{\mathbf{w}^{\mathrm{H}} \mathbf{w}}+j \overline{\mathbf{x}}^{(q)}\right),
\end{aligned}
$$

$$
\begin{aligned}
\nabla Q\left(g_{I}^{(q, a)}(\mathbf{w})\right)= & \frac{e^{-\frac{1}{2}\left(g_{I}^{(q, a)}(\mathbf{w})\right)^{2}}}{2 \sqrt{2 \pi} \sigma_{n} \sqrt{\mathbf{w}^{\mathrm{H}} \mathbf{W}}}\left(\frac{2 c_{1}+\operatorname{sgn}\left(b_{I_{1}}^{(q)}\right) \bar{y}_{I}^{(q)}}{\mathbf{w}^{\mathrm{H}} \mathbf{w}} \mathbf{w}\right. \\
& \left.+j \operatorname{sgn}\left(b_{I_{1}}^{(q)}\right) \mathbf{x}^{(q)}-2 \mathbf{p}_{1}\right) .
\end{aligned}
$$

Note that the BER is invariant to a positive scaling of $\mathbf{w}$. It is therefore computationally advantageous to normalise $\mathrm{w}$ to a unit-length, so that $\mathrm{w}^{\mathrm{H}} \mathbf{w}=1$ in the above gradient formulae (35) to (38). The optimisation problem (33) can be solved iteratively using a gradient-based algorithm. In particular, the following simplified conjugate gradient algorithm [7], [9] provides an efficient means to find an MBER solution.

Initialization. Choose a step size of $\mu>0$ and a termination scalar of $\beta>0$; given $\mathbf{w}(1)$ and $\mathbf{d}(1)=-\nabla P_{E}(\mathbf{w}(1))$; set the iteration index to $\iota=1$.

Loop. If $\left\|\nabla P_{E}(\mathbf{w}(\iota))\right\|<\beta$ : goto Stop. Else:

$$
\begin{gathered}
\mathbf{w}(\iota+1)=\mathbf{w}(\iota)+\mu \mathbf{d}(\iota) ; c_{1}=\mathbf{w}^{\mathrm{H}}(\iota+1) \mathbf{p}_{1} ; \\
\mathbf{w}(\iota+1)=\frac{c_{1}}{\left|c_{1}\right|} \mathbf{w}(\iota+1) ; \mathbf{w}(\iota+1)=\frac{\mathbf{w}(\iota+1)}{\|\mathbf{w}(\iota+1)\|} ; \\
\phi_{\iota}=\frac{\left\|\nabla P_{E}(\mathbf{w}(\iota+1))\right\|^{2}}{\left\|\nabla P_{E}(\mathbf{w}(\iota))\right\|^{2}} ; \mathbf{d}(\iota+1)=\phi_{\iota} \mathbf{d}(\iota)-\nabla P_{E}(\mathbf{w}(\iota+1)) ;
\end{gathered}
$$

then set $\iota=\iota+1$ and goto Loop.

Stop. $\mathbf{w}(\iota)$ is the solution.

At a minimum we have $\left\|\nabla P_{E}(\mathbf{w})\right\|=0$. Hence the termination scalar $\beta$ determines the accuracy of the solution obtained. The step size $\mu$ controls the rate of convergence. Typically, a much larger value of $\mu$ can be used compared to the steepest-descent gradient algorithm. As the BER surface $P_{E}(\mathbf{w})$ is highly nonlinear, occasionally the search direction d may no longer be a good approximation to the conjugate gradient direction or may even point to the "uphill" direction, when the iteration index becomes large. It is thus advisable to periodically reset $\mathbf{d}$ to the negative gradient in the above conjugate gradient algorithm. With this resetting mechanism, this simplified conjugate gradient algorithm has been shown to converge fast to the theoretical MBER solution, typically in tens to hundred iterations, in many simulation studies. By resetting $\mathbf{d}$ to the negative gradient every iteration, this algorithm reduces to the steepest-descent gradient algorithm.

\section{Simulation Study}

The simulated beamforming system consisted of four 16QAM user sources with a three-element linear uniform antenna array. The locations of the desired user and the interfering users were graphically shown in Fig. 2. All the four users were assumed to have an equal transmit power, and the four channel taps, $A_{i}$ for $1 \leq i \leq 4$, were identical. The interfering user 2 was the "heaviest" interferer, which had the minimum angular separation with the desired user, that is, $\theta<60^{\circ}$. In our simulation, the perfect channel knowledge was assumed at the receiver. The proposed MBER beamforming receiver was compared with the classical MMSE beamforming receiver as well as the MSER beamforming receiver of [7]. 


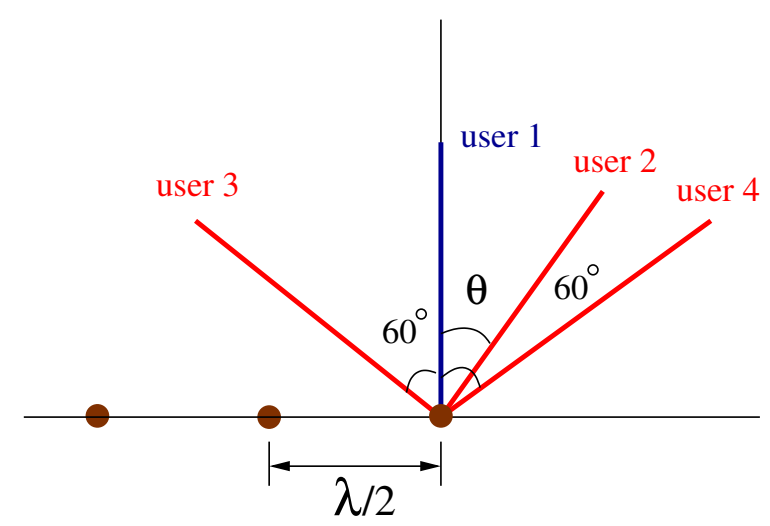

Fig. 2. Locations of the four 16-QAM users with respective to the threeelement receive antenna array having $\lambda / 2$ element spacing, where $\lambda$ represents the wavelength and $\theta$ denotes the minimum angular separation.

In the case of the minimum angular separation $\theta=38^{\circ}$, the BER performance achievable by the MMSE, MBER and MSER beamforming designs are compared in Fig. 3. As expected, both the MSER solution and the proposed MBER solution attained the same BER performance, and they significantly outperformed the conventional MMSE design. When the minimum angular separation was reduced to $\theta=35^{\circ}$, the MMSE beamforming could not cope with the heavy interference and its BER exhibited an error floor, as can be seen clearly from Fig. 4. By contrast, both the MBER and MSER designs were capable of operating satisfactorily in such a heavy interference senario.

\section{CONCLUSIONS}

We have proposed an MBER beamforming receiver for multi-user SDMA based QAM systems. The BER formula has been derived explicitly, and the optimal MBER solution has been obtained by minimising the BER cost function using

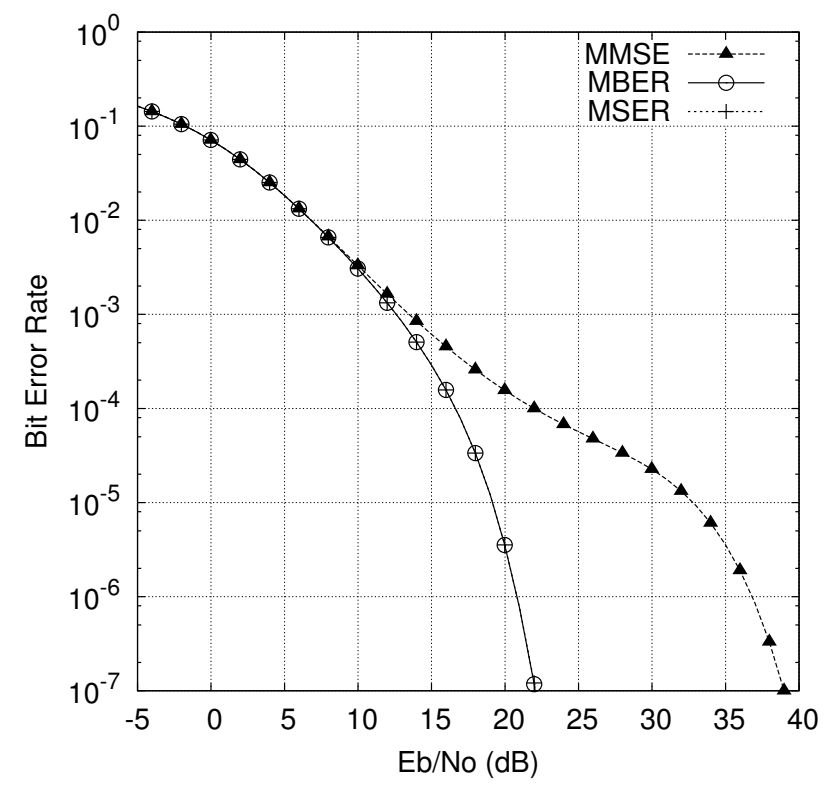

Fig. 3. Bit error rate performance comparison of three beamforming designs, where $\theta=38^{\circ}$

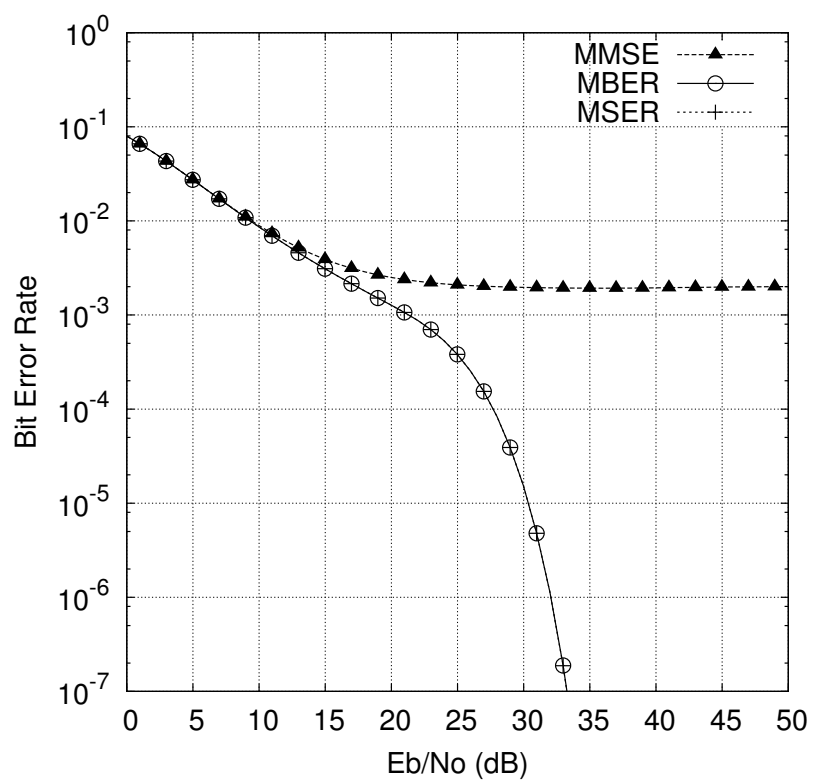

Fig. 4. Bit error rate performance comparison of three beamforming designs, where $\theta=35^{\circ}$.

an efficient simplified conjugate gradient algorithm. Although the bit decision procedure is inherently more complicated than the symbol decision making process, it is interesting to see that the BER calculation turns out to have a similar complexity as the SER calculation, at least for the 16-QAM case. Therefore, the MBER beamforming solution requires a similar computational complexity to that of the MSER solution. The simulation results obtained have also confirmed that the MBER beamforming receiver attains the same BER performance as the MSER beamforming receiver, and the both solutions significantly outperform the standard MMSE-based beamforming receiver.

\section{REFERENCES}

[1] J. Litva and T. K. Y. Lo, Digital Beamforming in Wireless Communications. London, UK: Artech, 1996.

[2] J. H. Winters, "Smart antennas for wireless systems," IEEE Pers. Commun., vol. 5, no. 1, pp. 23-27, Jan. 1998.

[3] J. S. Blogh and L. Hanzo, Third Generation Systems and Intelligent Wireless Networking - Smart Antennas and Adaptive Modulation. New York: Wiley, 2002.

[4] S. Chen, N. N. Ahmad, and L. Hanzo, "Adaptive minimum bit error rate beamforming," IEEE Trans. Wireless Communications, vol. 4, no. 2 pp. 341-348, March 2005.

[5] S. Chen, L. Hanzo, N. N. Ahmad, and A. Wolfgang, "Adaptive minimum bit error rate beamforming assisted receiver for QPSK wireless communication," Digital Signal Processing, vol. 15, no. 6, pp. 545-567, 2005.

[6] L. Hanzo, W. Webb and T. Keller, Single- and Multi-Carrier Quadrature Amplitude Modulation: Principles and Applications for Personal Communications, WLANs and Broadcasting. Chichester, UK: Wiley, 2000.

[7] S. Chen, A. Livingstone, H. Du, and L. Hanzo, "Adaptive minimum symbol error rate beamforming assisted detection for quadrature amplitude modulation," IEEE Trans. Wireless Communications, vol. 7, no. 4, pp. 1140-1145, April 2008.

[8] S. Haykin, Adaptive Filter Theory (third edition). Prentice Hall, Upper Saddle River, NJ.

[9] M. S. Bazaraa, H. D. Sherali, and C. M. Shetty, Nonlinear Programming: Theory and Algorithms Wiley, New York. 